\title{
ESTUDIO PRESUROMÉTRICO Y BIOMECÁNICO DEL PIE EN EL PÁDEL
}

\section{PRESSUROMETRY AND BIOMECHANICAL STUDY OF THE FOOT IN PADEL}

\author{
Priego, J.I. ${ }^{1}$, Olaso Melis, J. ${ }^{1}$, Llana-Belloch, S. ${ }^{1}$, Pérez-Soriano, P. ${ }^{1}$, González García, J.C. ${ }^{2}$, \\ Sanchís Almenara, M. ${ }^{2}$ \\ ${ }^{1}$ Grupo de Investigación Biomecánica aplicada al Deporte (GIBD), Departamento de Educación Física y Deportiva, \\ Universidad de Valencia, Facultat de Ciències de l'Activitat Física i l'Esport. C/ Gascó Oliag, 3. 46010. Valencia, España. \\ ${ }^{2}$ Instituto de Biomecánica de Valencia (IBV), Universidad Politécnica de Valencia, Camino de Vera s/n, 46022, Valencia, \\ España.
}

Jose Ignacio Priego Quesada, Licenciado en Ciencias de la Actividad Física y el Deporte e investigador no doctor contratado en la Universidad de Valencia, j.priego.gibd@gmail.com

Jose Olaso Melis, Doctor Ingeniero, pepitolaso@gmail.com

Salvador Llana Belloch, Doctor en Ciencias de la Actividad Física y el Deporte y profesor titular de la Universidad de Valencia, salvador.llana@uv.es

Pedro Pérez Soriano, Doctor en Ciencias de la Actividad Física y el Deporte y profesor titular de la Universidad de Valencia pedro.perez-soriano@uv.es

Juan Carlos González García, Doctor Ingeniero y responsable del departamento de Indumentaria en el IBV, juancarlos.gonzalez@ibv.upv.es

Mercedes Sanchís Almenara, Ingeniera industrial, investigadora en el IBV, mercedes.sanchis@ibv.upv.es

Código UNESCO: 2406.04 Biomecánica

Clasificación Consejo de Europa: 3. Biomecánica del deporte

Recibido el 30 de marzo de 2014

Aceptado el 11 de abril de 2014

PALABRAS
CLAVE:
Antepié,
Deporte,
Raqueta,
Presión plantar,
Split-step,
Calzado,
Entrenamiento

\section{KEY WORDS:}

Forefoot,

Sport,

Racket,

Plantar pressure

Split-step,

Footwear,

Training

\section{RESUMEN}

El pádel es un deporte muy practicado en países como España, Argentina y Brasil, pero existen pocos artículos científicos que muestren la lesividad de su práctica. Los movimientos más frecuentes del pádel pueden incidir de forma lesiva en el pie y en la articulación del tobillo. Por ello, el objetivo del presente estudio fue profundizar en el análisis del rol del pie en la ejecución de los movimientos más representativos del pádel mediante técnicas de video y de presurometría. El estudio fue dividido en dos partes: análisis presurométrico de dos gestos (carrera frontal y el split-step), y filmación del pié en el trascurso de dos partidos. Las principales conclusiones obtenidas del estudio son la importancia del antepié en los movimientos analizados, la importancia de un diseño específico del calzado para el pádel, y la importancia del entrenamiento neuromuscular y propioceptivo del complejo pie-tobillo, especialmente de los flexores plantares.

\section{ABSTRACT}

Padel is a very popular sport in countries like Spain, Argentina and Brazil, but there is a lack of scientific papers analyzing the harmfulness of their practice. Padel movements could potentially damage the foot and ankle joint. Therefore, the objective of this study was the characterization of the foot role during the execution of the most representative padel movements by techniques of pressurometry and video. The study was divided in two parts: pressurometric analysis of two movements (head-on displacement and split-step), and video recording of the foot during two games. The main conclusions of the study are the importance of forefoot zone in the padel movements, the importance of a specific shoe design for padel practice, and the importance of neuromuscular and proprioceptive training of the ankle and plantar flexors. 


\section{INTRODUCCIÓN}

En la última década, el pádel ha experimentado un gran crecimiento en países como España, Argentina y Brasil. Dos datos muestran de forma evidente este hecho. En primer lugar, el aumento del $101 \%$ de licencias de jugadores en España desde 1998 hasta $2011^{(1)}$. En segundo lugar, el hecho de que en Argentina sea el segundo deporte más practicado con hasta 4 millones de jugadores ${ }^{(2)}$. Este gran auge puede explicarse por ser un deporte muy atractivo para cualquier público debido a diferentes características como son el tamaño de la pista $^{(3)}$, la existencia de paredes que facilitan poder devolver la pelota ${ }^{(3)}$, y una exigencia fisiológica moderada ${ }^{(4)}$. Sin embargo, a pesar del gran crecimiento en el número de jugadores, debido a la juventud del pádel como deporte, existe poco conocimiento científico sobre la lesividad derivada de su práctica.

Hasta el momento no existe un estudio amplio que muestre la epidemiología en el pádel. García Acedo y Ares Bella ${ }^{(5)}$ señalaron que en su experiencia clínica, las tres lesiones que más suceden en el pádel son los esguinces de tobillo $(27.27 \%)$, las microrupturas de gastronemios $(18.18 \%)$ y las sesamoiditis y fascitis plantares (18.18\%). Estos datos pueden plantear la hipótesis de que debido a las características de los movimientos del pádel, el pié y la articulación del tobillo son las estructuras que más pueden sufrir durante la práctica deportiva. No obstante, ningún estudio ha analizado con detenimiento qué sucede con dichas estructuras durante la ejecución de los movimientos más representativos del pádel como son el desplazamiento lateral (con una frecuencia de 16.14 repeticiones cada 5 minutos de juego), la carrera frontal (13.36 repeticiones cada 5 minutos de juego), los split-steps (7.57 repeticiones cada 5 minutos de juego), los giros pivotando un pie (2.43 repeticiones cada 5 minutos de juego), y los golpeos ${ }^{(1)}$.

De este modo, el objetivo del presente estudio fue analizar cualitativamente el rol del pie durante la ejecución de los movimientos más representativos del pádel (mediante técnicas de video y de presurometría), para poder ofrecer una información relevante que permita definir mejor el entrenamiento, el diseño de calzado y las medidas para prevenir lesiones.

\section{MATERIAL Y MÉTODOS}

El estudio se dividió en dos partes: análisis mediante presurometría de dos gestos representativos del pádel como son la carrera frontal y el split-step, y filmación del pié en el trascurso de dos partidos de pádel.

\section{Participantes}

Participaron en el estudio diez jugadores de pádel (edad: $33 \pm 8,2$ años, peso: $78,4 \pm 3,7 \mathrm{~kg}$; altura: $177,7 \pm 2,6 \mathrm{~cm}$; horas semanales de práctica: $7,5 \pm 3,9$ horas; partidos a la semana: $3,2 \pm 1,2$ partidos; años de experiencia: $8,3 \pm 4,1$ años) sin lesiones en los 3 meses anteriores al estudio, y vistiendo el calzado que habitualmente usan para la práctica del pádel.

Los participantes firmaron un formulario de consentimiento informado antes de iniciar el protocolo, de acuerdo con la Comisión de Investigación de la Universidad Politécnica de Valencia (España). Todos los procedimientos cumplen con los requisitos que figuran en la Declaración de Helsinki de 1975 (y su modificación posterior en 2008) y están de acuerdo con las normas éticas de la revista International Journal of Sports Medicine ${ }^{(6)}$.

\section{Medidas}

\section{Análisis mediante presurometría}

Para el análisis cualitativo de las presiones plantares se utilizó el sistema Biofoot IBV (IBV,

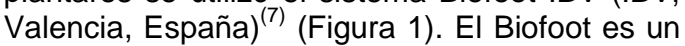
sistema de plantillas de sensores con el que se puede medir la evolución de las presiones durante la marcha o diferentes movimientos del pie.

Fueron registrados dos gestos en ambos pies: la carrera frontal y el split-step. Los 10 participantes realizaron 3 repeticiones de cada uno de los gestos intentando simular todo lo posible que se encontraban en una situación real de partido, a una percepción de intensidad moderada.

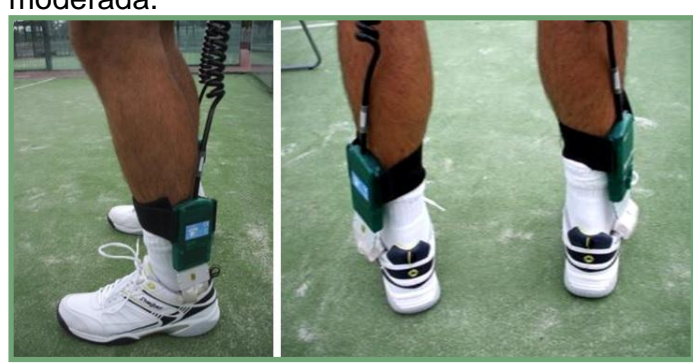

Figura 1. Análisis presurométrico mediante Biofoot en el pádel en ambos pies.

Se promediaron las presiones de plantares máximas de las 3 repeticiones de cada uno de los participantes en ambos pies, y a posteriori se promediaron las presiones de los 10 jugadores mediante un algoritmo implementado en MATLAB® (Mathworks Inc., Natick, Massachusetts, USA). De estos promedios se obtuvo una imagen de la distribución de presión plantar para cada uno de los tres gestos.

Rev. lb. Cᄃ. Act. Fis. Dep. 


\section{Filmación de dos partidos.}

Se realizó la grabación de dos partidos de pádel reales con 8 de los jugadores de la muestra seleccionados aleatoriamente. Cada partido tuvo una duración de una hora. La filmación se centró en las extremidades inferiores para conocer en profundidad con qué zonas del pié se realizan los apoyos de los diferentes movimientos. Se utilizó una cámara de video de alta definición (Sony Handycam HDR-FX1E, Sony Corp., Tokyo, Japan) con una frecuencia de muestreo de $55 \mathrm{~Hz}$, posicionada detrás de la pista, perpendicular a los pies de los jugadores y a una distancia de aproximadamente 5 metros de la línea de servicio.

Se visualizaron las dos horas totales de los dos partidos y se extrajo una valoración cualitativa de los movimientos más representativos ${ }^{(1)}$ : desplazamiento lateral, la carrera frontal, los split-steps, los giros pivotando sobre un pie, y los golpeos.

\section{RESULTADOS}

1. Análisis cualitativo mediante presurometría.

En la realización del gesto deportivo de carrera frontal, se obtuvieron mayores presiones en la zona media del antepié y en el final del retropié (Figura 2A). Aunque con menor presión que las anteriores, también se puede observar una mayor presión en la zona del primer dedo respecto al resto del pie (Figura $2 \mathrm{~A}$ ).

En la realización del gesto deportivo de splitstep, se puede ver que las presiones más altas se alcanzan en el antepié, especialmente en la zona media (Figura 2B). Se observa unas presiones muy bajas o casi nulas en el talón en el resto del pie (Figura 2B).

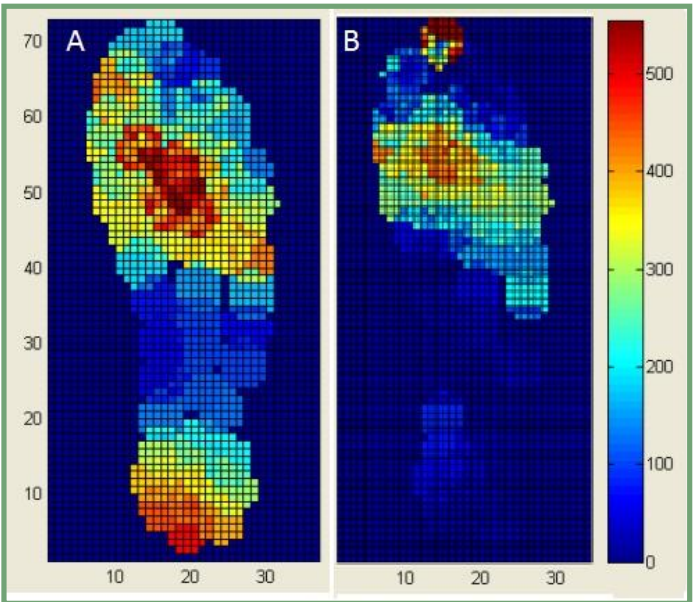

Figura 2. Mapa de presiones durante la realización de gestos en el pádel (A-carrera frontal, B- split-step)
2. Análisis cualitativo mediante la filmación de dos partidos.

Desplazamiento lateral. Se observó que durante el desplazamiento lateral la zona que realiza la mayor parte del apoyo es el antepié. Este tipo de movimiento se ve influenciado por la velocidad en la que se ejecuta. A una velocidad baja se suele apoyar el talón en el desplazamiento, mientras que en los movimientos más rápidos, que son la mayoría, los jugadores no apoyan el resto del pie, descargando todo el peso sobre las cabezas metatarsianas.

Carrera frontal. Al igual que en el desplazamiento lateral, la zona de presión en la carrera frontal variará según la velocidad del movimiento. Si consiste en unos pasos a una velocidad baja o moderada, el patrón motor es el típico de los corredores talonadores, es decir, el primer apoyo con el retropié y despegando con el antepié. Si por el contrario se realizan pasos en sprint, los jugadores limitan su apoyo al antepié.

Split-Step. Los jugadores realizan saltos de muy poca altura, numerosos y rápidos. Estos pequeños saltos son realizados completamente con el antepié, apoyando sobre las cabezas metatarsianas y los dedos del pie (Figura 3 ).

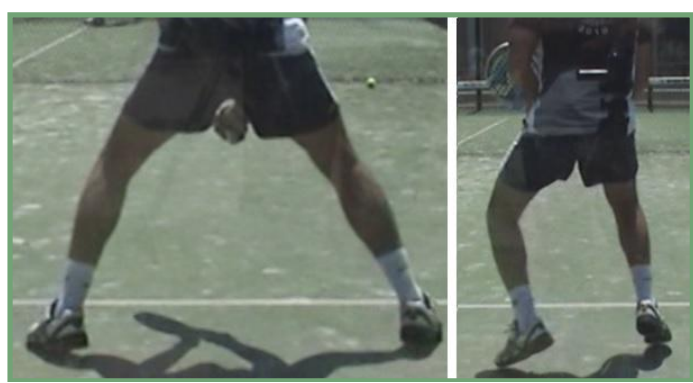

Figura 3. Ejemplos del comportamiento del pie durante el Split-step.

Giros de pivote. Los jugadores realizan la mayoría de los giros de pivote con el antepié como punto de apoyo para realizar la rotación (Figura 4A). Por otro lado se observó que en algunas ocasiones, diferentes jugadores realizaban el pivote de rotación con el retropié (Figura 4B).

Rev. Ib. Cᄃ. Act. Fis. Dep. 


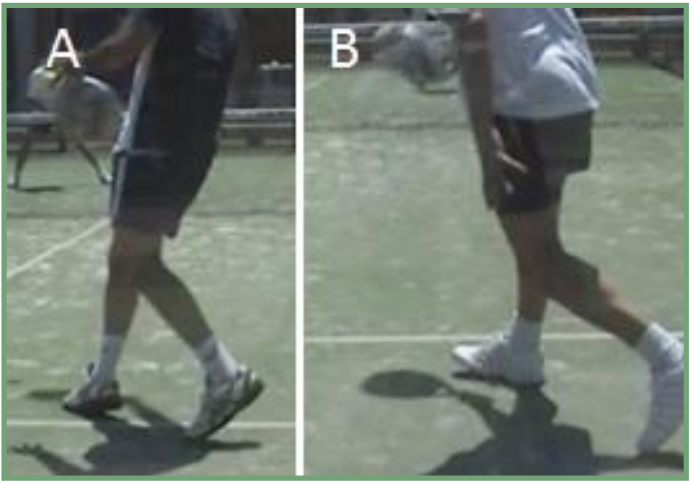

Figura 4. Ejemplos del comportamiento del pie durante los giros de pivote (A-realizando el giro con el antepié, B- realizando el giro con el retropié).

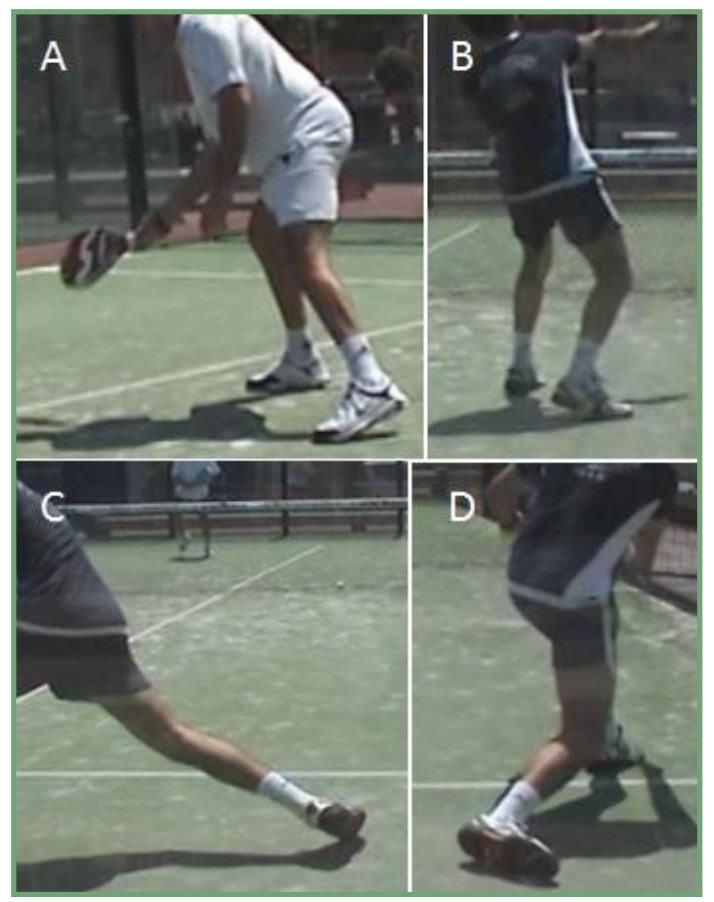

Figura 5. Ejemplos del comportamiento del pie en diferentes tipos de golpeos (A-golpeo cómodo de derecha, B- remate, C- golpeo forzado con arrastre del antepié anterior, Dgolpeo forzado con arrastre del antepié anterior y medial).

Golpeos. El comportamiento del pie durante el golpeo dependerá de la dificultad de su ejecución. Cuando el jugador realiza cómodo el golpeo por no haberse desplazado o haberse podido parar mucho antes de su realización, se observa que uno de los pies se mantiene completamente apoyado, mientras que el otro levanta el talón manteniendo el apoyo sobre los dedos (Figura 5A). En el caso de golpeos más forzados, se ha observado que en el instante del golpeo, uno de los pies suele fijarse mientras que el otro realiza un desplazamiento de arrastre con la zona del antepié anterior y/o medial (Figura 5C y 5D). Por otro lado, se ha observado que en los remates, se parte de un apoyo completo de los dos pies para llegar a mantenerse de puntillas en el remate en ambos pies (Figura 5B).

\section{DISCUSIÓN}

Los diferentes análisis realizados en el presente estudio apoyan que el antepié es la zona del pie que soporta mayores presiones en el pádel. En la filmación con video se ha observado que en todos los movimientos analizados (desplazamiento lateral, carrera frontal, splitstep y giros de pivote) es la zona con la que se realiza la mayor parte del apoyo plantar. Esto se ha corroborado con el análisis presurométrico de la carrera frontal y el split-step donde se han encontrado mayores presiones en dicha zona. Esto es importante a la hora de prevenir lesiones debido a que sobrecargas de presión plantar han sido relacionadas con lesiones como sesamoiditis, fascitis, y fracturas por esfuerzo entre otras ${ }^{(8,9)}$ y tanto en el pádel como en los deportes de raqueta de modo general, son consideradas lesiones muy comunes ${ }^{(5,10)}$. Por lo tanto, esto debería ser tenido en cuenta a la hora de diseñar calzado específico para pádel, proporcionando una amortiguación suficiente para esta zona, y una buena flexibilidad en la zona media del pie que permita el correcto desacoplamiento del antepié y el retropié en los diferentes movimientos. También debe ser un aspecto a tener en cuenta por los entrenadores a la hora de planificar las cargas de trabajo.

Al igual que en el tenis ${ }^{(11-14)}$, el desplazamiento lateral tiene una la gran importancia en el pádel al ser el movimiento más frecuente ${ }^{(1)}$. El presente estudio ha mostrado que la velocidad a la que se ejecute el desplazamiento lateral afecta a su patrón motor, haciendo que en desplazamientos laterales rápidos se produzca un apoyo único del antepié, mientras que a velocidades más bajas se distribuya el peso en una mayor superficie.

El split-step es un movimiento preparatorio realizado antes del golpeo del oponente y que permite acortar los tiempos de reacción en movimientos posteriores ${ }^{(15,16)}$. Diferentes estudios han observado que con el split-step se consiguen movimientos posteriores más rápidos y con un menor tiempo de reacción que sin él $^{(16,17)}$. Uzu et al. ${ }^{(17)}$ observaron que el splitstep provoca una gran activación del gastrocnemius medialis (gemelo interno), mientras que Nieminen et al. ${ }^{(16)}$ encontraron una gran actividad en el soleo. El presente estudio muestra que los split-steps se realizan apoyando el antepié, y por lo tanto es una acción mediada por los flexores plantares (también llamados extensores del tobillo), entre

Rev. lb. Cᄃ. Act. Fis. Dep. 
los que destacan el soleo y los gastrocnemios. Teniendo en cuenta que García Acedo y Ares Bella $^{(5)}$ observaron una gran frecuencia de microrupturas de gastronemios en el pádel, que el split-step ha sido determinado como un gesto muy frecuente ${ }^{(1)}$, y que la mayoría de los movimientos en el pádel al estar realizando un apoyo del antepié están haciendo un uso de estos músculos, se evidencia una necesidad de que los jugadores de pádel entrenen bien esta musculatura con el fin de que no se fatigue $y$ prevengan posibles lesiones.

En relación a los giros de pivote, se ha observado que la mayoría de los giros se realizan teniendo como punto de apoyo el antepié, pero en algunos casos los jugadores utilizan el retropié como pivote de rotación. El dibujo de la suela del calzado tiene una gran efecto sobre la tracción ${ }^{(18)}$, y por lo tanto debe permitir este tipo de movimientos para evitar posibles lesiones de miembro inferior.

Durante los golpeos, el patrón analizado sugiere que los jugadores utilizan el pie de apoyo como pivote y el otro pie lo regulan con un apoyo parcial para alcanzar la posición de golpeo cómoda sin perder estabilidad. Además, el jugador puede optar por realizar intencionalmente un deslizamiento controlado del pie en movimiento. Durante estos movimientos, el lado medial (interno) de la suela de la zapatillas es la que impacta primero sobre el suelo, produciendo una gran palanca en relación con el eje de la articulación subastragalina $^{(19)}$, que si es excesiva, puede producir daño en los ligamentos laterales de la articulación del tobillo ${ }^{(20)}$. Dado que en el tenis también es muy común este tipo de deslizamiento lateral ${ }^{(21)}$ y los ligamentos del tobillo son muy proclives a sufrir lesiones ${ }^{(22)}$ al igual que parece que sucede en el pádel ${ }^{(5)}$, este movimiento debe ser tenido muy en cuenta.

Para reducir en lo posible el riesgo de lesión de los deslizamientos laterales, se puede actuar sobre diferentes focos:

1. El calzado. El éxito de este tipo de movimiento dependerá de la tracción dinámica desarrollada por el tipo de suela, y por lo tanto la suela deberá garantizar unos niveles de tracción laterales óptimos ${ }^{(21)}$. Tracciones excesivas de la suela son más proclives a producir lesiones a los jugadores ${ }^{(21)}$. Por otro lado, la magnitud de la palanca que sucede sobre la articulación astragalina, depende del diseño y las propiedades de la suela del zapato ${ }^{(19,23)}$. Según Stacoff et al. ${ }^{(19)}$ para reducir el riesgo de lesiones en este tipo de movimiento, el calzado debe reducir su inversión, así como la del pié dentro del calzado. Propiedades del calzado como unas características óptimas de dureza y torsión de la suela, así como una altura de caña adecuada, conseguirán una mayor estabilidad lateral y por tanto una menor inversión del pie ${ }^{(19)}$.

2. Niveles adecuados de propiocepción del jugador. Tener una buena propiocepción de la inversión del tobillo está asociado con un menor riesgo de tener lesiones en esta articulación ${ }^{(24-26)}$. Parece ser, que aunque existe un gran factor genético, cuanto mayor es el tiempo de entrenamiento de una persona en su deporte mayor es su capacidad propiceptiva ${ }^{(27,28)}$. Esto podría indicar que jugadores menos expertos, podrían tener una mayor probabilidad de sufrir lesiones de tobillo, con lo que podría ser aconsejable que estos deportistas realizasen entrenamiento específico neuromuscular y propioceptivo para disminuir el riesgo de lesión ${ }^{(26,29,30)}$.

Por último, se debe tener en cuenta que la fricción provocada por los deslizamientos laterales producirá un mayor desgaste de la suela y el material de corte en el lado interno de la parte delantera de la zapatilla. Debido a esto, el calzado deberá incorporar refuerzos en dicha zona con el objetivo de disminuir el desgaste.

\section{CONCLUSIONES}

Las conclusiones del presente estudio son las siguientes:

1. El antepié es la zona con la que se realiza la mayor parte del apoyo en los movimientos más frecuentes del pádel. Un diseño del calzado específico para pádel puede ser esencial a la hora de prevenir lesiones en esta zona.

2. Se deben de entrenar correctamente los músculos flexores plantares en el pádel, para evitar su fatiga y reducir la probabilidad de lesión.

3. El diseño del calzado debe permitir movimientos importantes en el pádel como son los desplazamientos laterales y los giros de pivote con el antepié.

4. Los deslizamientos laterales de uno de los pies durante el golpeo son muy comunes. Este tipo de acción puede ser lesiva y debe ser tenida en cuenta tanto en el diseño del calzado, como por los entrenadores, programando de forma adecuada el entrenamiento neuromuscular y propioceptivo del complejo pie-tobillo.

Rev. Ib. CL. Act. Fis. Dep. 


\section{REFERENCIAS BIBLIOGRÁFICAS}

1. Priego Jl, Olaso J, Llana-Belloch S, PérezSoriano P, García JCG, Sanchís MS. Padel: a quantitative study of the shots and movements in the high-performance. J Hum Sport Exerc. 2013;8(4).

2. Martínez BJS-A. Historia del Pádel= History of Padel. Mater Para Hist Deporte. 2013;(11):5760 .

3. Lasaga Rodríguez MJ. Estudio social y metodológico del Pádel desde la percepción de técnicos y jugadores: una apuesta educativa [Tesis doctoral]: Universidad de Sevilla; 2011.

4. Amieba C, Salinero JJ. Overview of paddle competition and its physiological demands. AGON Int J Sport Sci. 3 (2):60-7.

5. García Acedo DM, Ares Bella F. Estudio biomecánico y patomecánico en la práctica del padel. Rev Esp Podol. 2007;(4):176-83.

6. Harriss DJ, Atkinson G. International Journal of Sports Medicine-ethical standards in sport and exercise science research. Int J Sports Med. 2009;30(10):701-2.

7. García-Pérez JA, Pérez-Soriano $P$, Llana $S$, Martínez-Nova A, Sánchez-Zuriaga D. Effect of overground vs treadmill running on plantar pressure: Influence of fatigue. Gait Posture. 2013 Sep;38(4):929-33.

8. Hennig EM, Milani TL. In-Shoe Pressure Distribution for Running in Various Types of Footwear. J Appl Biomech. 1995;11(3).

9. Pérez $P$, Llana $S$, Encarnación A, Fuster MA. Marcha nórdica: actividad física alternativa en el cuidado del pie. Mot Eur J Hum Mov. 2010 Sep 27;22(0):83-94.

10. Bouché RT. Racquet Sports: Tennis, Badminton, Squash, Racquetball, and Handball. Athletic Footwear and Orthoses in Sports Medicine. Springer; 2010. p. 215-23.

11. Kovacs MS. Movement for Tennis: The Importance of Lateral Training. Strength Cond J. 2009 Aug;31(4):77-85.

12. Weber K, Pieper S, Exler T. Characteristics and significance of running speed at the Australian Open 2006 for training and injury prevention. Med Sci Tennis. 2007;12(1):14-7.

13. Llana-Belloch $S$, Brizuela G, Pérez-Soriano P, García-Belenguer AC, Crespo M. Supination control increases performance in sideward cutting movements in tennis. Sports Biomech. 2013;12(1):38-47.

14. Llana Belloch S, Brizuela Costa GA, Pérez Soriano P, García Berenguer AC. Factores de rendimiento en el desplazamiento del tenista influenciados por el calzado. Rev. int. cienc. deporte (RICYDE) 2009; 15: 81-94.

15. Aviles C, Benguigui N, Beaudoin E, Godart F. Developing early perception and getting ready for action on the return of serve. ITF Coach Sport Sci Rev. 2002;28:6-8.

16. Nieminen MJJ, Piirainen JM, Salmi JA, Linnamo V. Effects of neuromuscular function and split step on reaction speed in simulated tennis response. Eur J Sport Sci. 2013 Apr 20;
17. Uzu R, Shinya M, Oda S. A split-step shortens the time to perform a choice reaction step-andreach movement in a simulated tennis task. J Sports Sci. 2009 Oct;27(12):1233-40.

18. Ura D, Clarke J, Carré M. Effect of shoe orientation on shoe-surface traction in tennis. Footwear Sci. 2013;5(sup1):S86-S87.

19. Stacoff A, Steger J rg, St ssi E. Lateral stability in sideward cutting movements. J Biomech. 1994;27(6):690-690.

20. Wright IC, Neptune RR, van den Bogert AJ, Nigg BM. The influence of foot positioning on ankle sprains. J Biomech. 2000 May;33(5):513-9.

21. Clarke J, Dixon SJ, Damm L, Carré MJ. The effect of normal load force and roughness on the dynamic traction developed at the shoesurface interface in tennis. Sports Eng. 2013;16(3):165-71.

22. Abrams GD, Renstrom PA, Safran MR. Epidemiology of musculoskeletal injury in the tennis player. $\mathrm{Br} J$ Sports Med. 2012;46(7):492-8.

23. Stacoff A, Denoth J, Kaelin X, Stuessi E. Running Injuries and Shoe Construction: Some Possible Relationships. Int J Sport Biomech. 1988;4(4).

24. Witchalls J, Blanch $P$, Waddington G, Adams R. Intrinsic functional deficits associated with increased risk of ankle injuries: a systematic review with meta-analysis. $\mathrm{Br} \mathrm{J}$ Sports Med. 2012 Jun 1;46(7):515-23.

25. Han J, Waddington G, Anson J, Adams R. Level of competitive success achieved by elite athletes and multi-joint proprioceptive ability. $J$ Sci Med Sport Sports Med Aust. 2013 Dec 12;

26. Malliou VJ, Beneka AG, Gioftsidou AF, Malliou PK, Kallistratos E, Pafis GK, et al. Young tennis players and balance performance. $\mathrm{J}$ Strength Cond Res Natl Strength Cond Assoc. 2010 Feb;24(2):389-93.

27. Aydin T, Yildiz Y, Yildiz C, Kalyon TA. Effects of extensive training on female teenage gymnasts' active and passive ankle-joint position sense. J Sport Rehabil. 2002;11(1):110.

28. Muaidi QI, Nicholson LL, Refshauge KM. Do elite athletes exhibit enhanced proprioceptive acuity, range and strength of knee rotation compared with non-athletes? Scand J Med Sci Sports. 2009;19(1):103-12.

29. Verhagen EALM, Bay K. Optimising ankle sprain prevention: a critical review and practical appraisal of the literature. $\mathrm{Br} \mathrm{J}$ Sports Med. 2010 Dec;44(15):1082-8.

30. Micheo W, Baerga L, Miranda G. Basic principles regarding strength, flexibility, and stability exercises. PM R. 2012 Nov;4(11):805-11.

Referencias totales citadas: 30 .

Referencias citadas correspondientes a la Rev Ib CC Act Fis Dep: 0.

Rev. lb. Сᄃ. Act. Fis. Dep. 